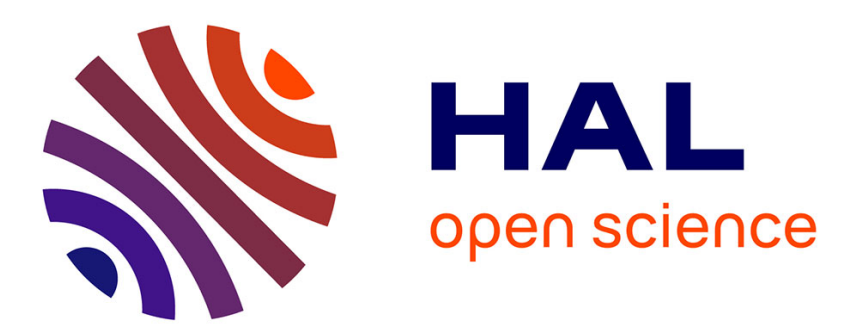

\title{
Numerical simulation of the fluid-structure interaction in a tube array under cross flow at moderate and high Reynolds number
}

\author{
V. Shinde, T. Marcel, Yannis Hoarau, T. Deloze, Gilles Harran, F. Baj, J. \\ Cardolaccia, J.P. P Magnaud, E. Longatte, Marianna Braza
}

\section{To cite this version:}

V. Shinde, T. Marcel, Yannis Hoarau, T. Deloze, Gilles Harran, et al.. Numerical simulation of the fluid-structure interaction in a tube array under cross flow at moderate and high Reynolds number. Journal of Fluids and Structures, 2014, 47, pp.99-113. 10.1016/j.jfluidstructs.2014.02.013 . hal02860426

\section{HAL Id: hal-02860426 \\ https://hal.science/hal-02860426}

Submitted on 19 Aug 2020

HAL is a multi-disciplinary open access archive for the deposit and dissemination of scientific research documents, whether they are published or not. The documents may come from teaching and research institutions in France or abroad, or from public or private research centers.
L'archive ouverte pluridisciplinaire HAL, est destinée au dépôt et à la diffusion de documents scientifiques de niveau recherche, publiés ou non, émanant des établissements d'enseignement et de recherche français ou étrangers, des laboratoires publics ou privés. 


\title{
Numerical simulation of the fluid structure interaction in a tube array under cross flow at moderate and high Reynolds number
}

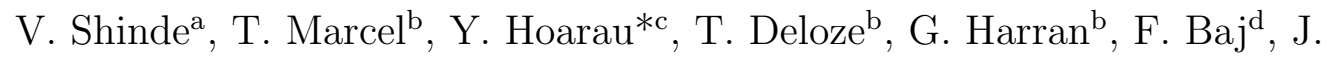 \\ Cardolaccia $^{\mathrm{d}}$, J.P. Magnaud ${ }^{\mathrm{d}}$, E. Longatte ${ }^{\mathrm{a}}$, M. Braza ${ }^{\mathrm{b}}$ \\ ${ }^{a}$ Laboratoire de Mécanique des Structures Industrielles Durables, LaMSID - UMR EDF \\ CNRS 2832, France \\ ${ }^{b}$ Institut de Mécanique des Fluides de Toulouse, IMFT- UMR CNRS 5502, France \\ ${ }^{c} I C U B E$, Université de Strasbourg, UMR CNRS 7357, France \\ ${ }^{d}$ Commissariat à l'Énergie Atomique (CEA), France
}

\begin{abstract}
The unsteady loads in a tube bundle are studied at moderate and high Reynolds number by means of URANS and hybrid (DDES) modelling. The onset of fluid-elastic instability is analysed for different structural parameters, Scruton number and reduced velocity. The simulations have been carried out with the code NSMB (Navier-Stokes Multi Block) by using turbulence modelling methods URANS and DDES (Delayed Detached Eddy Simulation). The CEA-DIVA configuration is considered for the cylinders array for an inter-tube Reynolds number 60,000. The study is carried out for a configuration of $(4 \times 5)$ cylinders in static conditions as well as for the vertical free motion of one of the central cylinders in one DOF (Degree Of Freedom).The inter-tube Reynolds number is 60,000. It is found that this cylinder spontaneously displays an oscillatory motion which first corresponds to Vortex Induced Vibration (VIV), associated to a lock-in mechanism for low values of the reduced velocity and secondly develops Movement Induced Vibration, MIV for higher values of the reduced velocity. The variation of the cylinder's oscillations frequency, of the unsteady loads and the structure's displacement are studied as a function of the reduced velocity for low and high values of the Scruton number. The increase of the phase-lag between the forces and the
\end{abstract}

\footnotetext{
*Corresponding author.

E-mail address: hoarau@unistra.fr.
} 
displacement is predicted and discussed for different Scruton number values and reduced velocities.

Keywords: 1 DOF Fluid-structure interaction, Cylinder array, Turbulent flow, Numerical simulation

\section{Introduction}

The prediction of fluid-elastic instabilities developed in a tube bundle is of major importance for the design of heat exchangers for vapor cooling in nuclear reactors and for the prevention of accidents associated with material fatigue, shocks between beams and severance of the solid walls. The fluid-elastic instabilities leading to flutter in tube-arrays appear in the laminar regime and persist as far as the turbulent regime.This kind of galloping instability has been identified for the past forty years. However, little has been achieved in the domain of detailed numerical simulations for the prediction of the unsteady loads and of the displacement of the solid structure. Many studies have been devoted to enhancing understanding and to offer phenomenological models for the design. A considerable number of this kind of models have been developed since the work of Connors (1970), Blevins (1974), Blevins (1979), Tanaka and Takahara (1981), Tanaka and Takahara (1982), Chen (1983), Paidoussis and Price (1988), Lever and Weaver (1982) among others.

Explaining and understanding the instability onset and its dependence on the flow-structure parameters such as the reduced velocity and Scruton number increase, is an important aspect concerning fundamental phenomena in the domain of fluid-structure interaction. A key point for the understanding of this instability is related to the appearance of negative damping which comes from the fluid forces interacting with the structure. This occurs when the transverse flow velocity increases, where the phase difference between the force applied by the fluid and the cylinder's movement changes sign and results in a sign change of the apparent damping, thereby creating fluid-elastic instability. The main problem is to correctly evaluate this phase shift model. Despite various modelling attempts, the question remains open, as noted by Weaver (2008) : "Despite more than 40 years of research, this mechanism is not fully understood". However quasi-static models are used to characterize the instability in high Scruton numbers Scruton $(\mathrm{Sc}>30)$ and in two or more degrees of freedom. For low Scruton numbers, the instability 
develops already for a single degree of freedom. This phenomenon can be modeled by taking into account a delay time between the movement and the efforts. The pure delay model is "amnesia." Granger and Paidoussis (1996) proposed a model "with memory" of first or second-order in respect of the time constants.

In the industrial context, the high Reynolds number causes a complex interaction between the instability due to the movement of the solid structure and the near-wall unsteady turbulence around the cylinders. To take sufficient account of this interaction and to accurately predict the unsteady loads, it is necessary to use reliable turbulence modelling approaches. These methods have to simultaneously include the low frequency organised motion effects associated with the structure's movement and the fluid's coherent vortices as well as the random turbulence effects. In this context, approaches such as Unsteady Reynolds Average Navier-Stokes (URANS), derived from turbulence in statistical equilibrium assumptions, tend to underestimate the global coefficients (drag, lift) and their amplitudes (see collected papers after the European research program "Detached Eddy Simulation for Industrial Aerodynamics" (DESIDER), Haase et al. (2009)).

The Large Eddy Simulation (LES) is appropriate and offers a rich physical content in the moderate Reynolds number range. However, this approach is mandatorily 3-D and quite costly for design purposes at this stage. On the other hand, in higher Reynolds numbers it becomes more difficult to apply it by using realistic grid sizes, in respect of industrial design requirements. In this flow category, it is noticeable that the upstream nominal Reynolds number based on the free-stream velocity corresponds to a much higher inter-tube Reynolds number which can be three or four times higher than the nominal Re, depending on the pitch distance. Therefore, a 'moderate' upstream Reynolds number flow for the cylinders array corresponds to a high - Reynolds range in the flow physics context. These facts have to be considered for the CFD method choice in the Computational Fluid Dynamics-Computational Structural Mechanics (CFD-CSM) coupling.

Hybrid RANS-LES methods are quite suitable for this category of fluidelastic instability problems, because they associate the benefits of URANS in the near-region and those of LES in the regions of flow detachment. In particular, the Delayed Detached Eddy Simulation (DDES), Spalart et al. (2006) is a hybrid method which is successfully used for strongly detached unsteady flows as reported by the collected papers of the fourth 'Hybrid RANS-LES Methods' (HRLM) symposium, Fu et al. (2012). DDES can be 
considerably improved by using adapted URANS modelling in the near-wall region and adapted LES modelling in the flow detachment areas, in order to allow for modification of the turbulent scales accounting for non-equilibrium turbulence. In this context, improved approaches can be used to take account of these effects, as for example the Scale Adaptive Simulation (SAS), Menter and Egorov (2005), Menter and Bender (2003), the Organised Eddy Simulation (OES), Braza et al. (2006), Bourguet et al. (2008) among others. SAS adapts the Kolmogorov turbulence scale according to flow regions governed by non-equilibrium turbulence effects. OES accounts for stress-strain directional misalignment in non-equilibrium turbulence regions thanks to a tensorial eddy-viscosity concept derived from Differential Reynolds Stress Modelling (DRSM) projection on the principal directions of the strain-rate tensor.

The current efforts in turbulence modeling are devoted to accurately reproduce the flow physics in respect of instability amplification, of strong flow detachment and to accurately predict the associated frequencies and unsteady load fluctuations. Although a significant progress has been accomplished in the last decade, there still remain open questions with regard to the prediction of the above mechanisms with the accuracy required by the design and in particular of these mechanisms modification due to the fluid-structure coupling. To our knowledge, there do not exist predictions of vibration instability in the high-Reynolds number range by using efficient CFD and producing new results in this area. In particular, the progressive development and assessment of the phase-lag between the forces and the displacement of the solid structure from the Vortex Induced Vibration (VIV) state towards the Movement Induced Vibration (MIV) dynamics are among main objectives of the present study. Therefore, the present paper mainly focuses on new results obtained by means of efficient CFD with regard to the physics of Flow Induced Vibration (FIV) in a cylinders bundle in a Reynolds number range corresponding to industrial applications. An exhaustive comparison of turbulence models is not presented in this article. Only selected models from the previously mentioned state-of-the-art, able to deal with strong unsteadiness, with high-Reynolds number effects and with predominant instabilities, are considered and coupled with efficient numerical schemes. The purpose of a first part of the present study is to select among these few models, the most appropriate for the static-case unsteady flow simulations, in order to use them in the dynamic case simulations of FIV (central cylinder allowed for one degree of freedom vertical motion). Furthermore, a special attention 
is paid to examine the predictive capabilities of the finally selected modelling in two- and three-dimensions for the dynamic case at high Reynolds number. In the physical reality, the 2-D and 3-D mechanisms act simultaneously and it is difficult to distinguish those of the physical mechanisms having a two-dimensional origin from those which are three-dimensional. This can be accomplished by means of the numerical simulation. Moreover, it is im-

portant for the industrial design to assess the part of realiability allowing for two-dimensional predictions comparing to the full three-dimensional approach, which is much more costly. According to these elements, the present article is structured in the following sections: the mechanisms of fluid-elastic instability are studied for the configuration described in section 2, by using fluid-structure coupling where the CFD part involves URANS and DDES modelling as described in the same section. The motion of the solid structure is coupled with the fluid by means of the acceleration equation involving mass, damping and stiffness. Modal structural analysis is not needed in this context, because the solid remains rigid. The mesh is adapted to follow the cylinder's motion by means of the Arbitrary Lagrangian-Eulerian approach, ALE. The results are presented in section 3 and 4 . Section 3 refers to the static case of cylinder's bundle. The 'DIVA' configuration is studied at an inter-tube Reynolds number $R e=60,000$. This section allows for validation of the numerical method and of the turbulence modelling to be used for the dynamic case, as well as for a physical analysis of the flow instabilities occurring in the present Reynolds number range. Section 4 studies the fluidstructure interaction in case of 1 DOF motion, carried out by URANS (in 2-D) and hybrid URANS-LES (DDES) turbulence modelling in 3-D at the same Reynolds number value.

\section{Flow-structure configuration, numerical method and turbulence modelling}

\subsection{Flow-structure configuration}

The fluid-structure configuration corresponds to an experimental set-up (DIVA) designed by the CEA where 20 cylinders are arranged in 5 columns with a pitch of $1.5 D, D$ being the cylinder's diameter. The upstream Reynolds number is of 20000 which corresponds to an inter-tube Reynolds number of 60 000. The 2-D grid is composed by $\sim 400000$ cells distributed in 80 structured blocs. 
The first grid distance from the wall is located at the distance $1,5.10^{-4} \mathrm{D}$ which assures a $y^{+}$value lower than 1 , as required by the turbulence modelling assumptions used in the present study, according to synthesis of works collected by Haase et al. (2009) and Fu et al. (2012). The spanwise length of this grid is 1D and 50 cells have been used in the $\mathrm{z}$ direction resulting in a grid of $\sim 23 M$ cells and 8192 blocks.

This mesh has been derived according to detailed grid-dependence studies concerning the refinement near the wall, (Skopek et al. (2012)), as well as in the PhD thesis of Marcel (2011), in order to provide grid-independent results. The dimensionless time step $\Delta t=d t * U_{\infty} / D$ used for the static configuration is $10^{-3}$, according to the numerical study by Ferreira-Perez (2013), providing time-step independence of the solution. In the following, $t^{*}$ designates the dimensionless time, $t^{*}=t * U \infty / D$. The influence of the time step is presented in the present article in the section of the dynamic motion.

The spanwise length of this grid is $1 D$ and 50 cells have been used in this direction. The domain size is presented in figure 1. The inflow distance is $10 D$, the outflow distance is $12 D$, the upper and lower boundary distances from the central cylinder are 3-D.

The boundary conditions are presented in the following :

- the inlet velocity is uniform and a free-stream turbulence level of $0.1 \%$ has been used.

- At the outlet boundary : subsonic outflow with imposed static pressure based on Riemann invariants.

- top-bottom boundaries in the $(x, y)$ plane : no-slip walls.

- spanwise boundary conditions : symmetry and periodic conditions have been compared. The symmetry boundary conditions have been chosen in order to not force a preferential spanwise wavelength.

\subsection{Numerical method}

The simulations have been performed with the Navier-Stokes Multi-Block $(N S M B)$ solver. The NSMB solver is the fruit of a european consortium that included Airbus from the beginning of '90's, as well as main European aeronautics research Institutes, as KTH, EPFL, IMFT, ICUBE, CERFACS, 

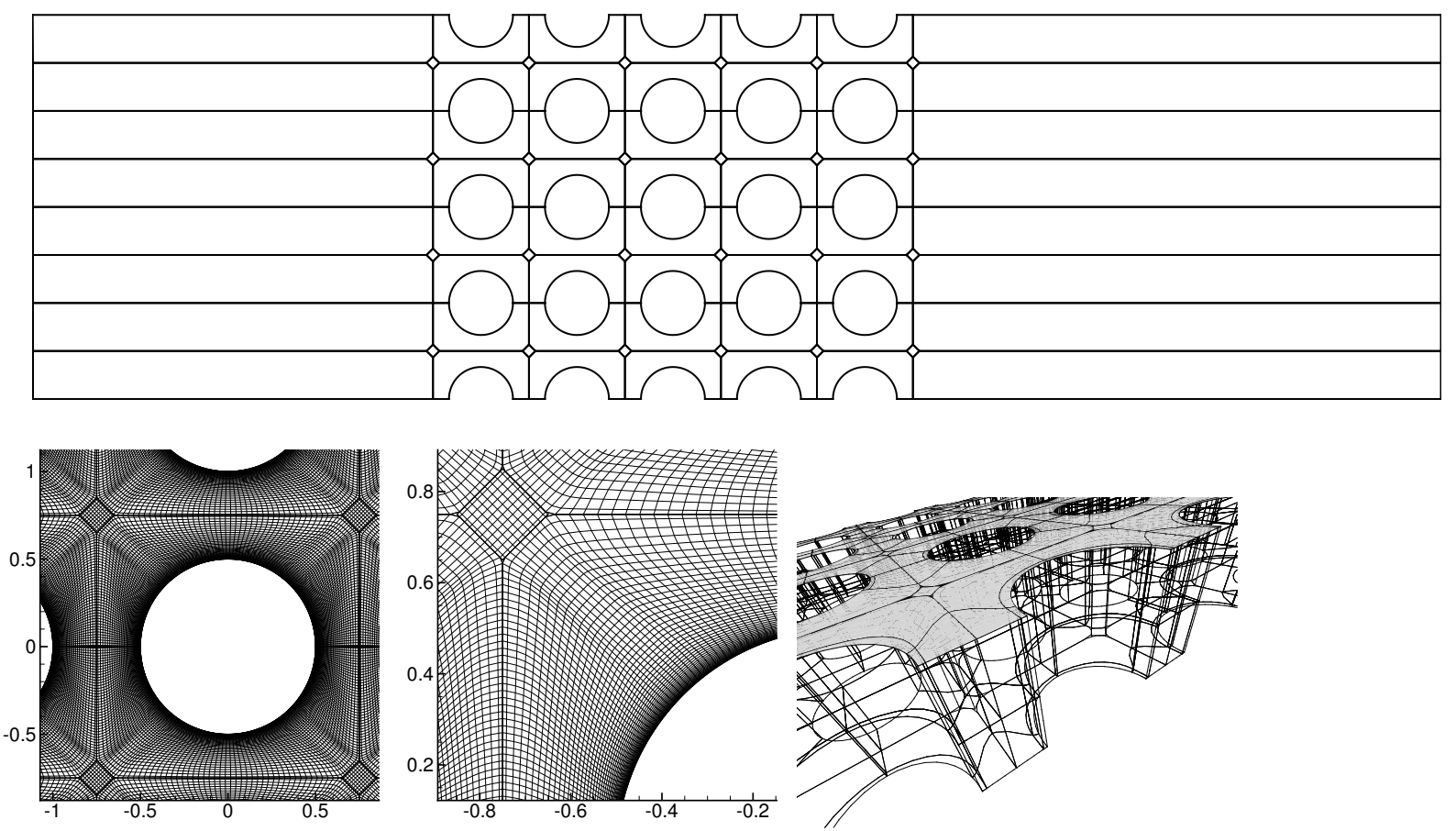

Figure 1: Grid overview and computational domain

Univ. of Karlsruhe, ETH-Ecole Polytechnique de Zurich, among other. This consortium is coordinated by CFS Engineering in Lausanne, Switzerland. NSMB is a structured code including a variety of efficient high-order numerical schemes and turbulence modelling closures in the context of LES, URANS and RANS-LES hybrid turbulence modelling, especially DDES (Delayed Detached Eddy Simulation). A first reference of the code description can be found in Vos et al. (1998) concerning the versions of this code in the decade of '90's. Since then, NSMB highly evolved up to now and includes an ensemble of the most efficient CFD methods, as well as efficient fluid-structure coupling for moving and deformable structures. These developments can be found in Hoarau (2002) regarding URANS modelling for strongly detached flows, Martinat et al (2008), in the area of moving body configurations, Barbut et al (2010), Grossi et al. (2014) allowing for Detached Eddy Simulation with the NSMB code.

The solid structure motion is taken into account by the Arbitrary La- 
grangian Eulerian method (ALE). The current version of NSMB code includes CHIMERA grid methods, Deloze et al. (2010), to handle very complex geometries and body motion and deformation. NSMB solves the compressible Navier-Stokes equations using a finite volume formulation on Multi-Block structured grids. Furthermore, this code includes different preconditioning numerical schemes to solve very low Mach number / incompressible flows. The time integration relies on a second-order backward Euler scheme based on the full matrix implicit LU-SGS (Lower-Upper Symmetric Gauss-Seidel) method and on the dual-time stepping, performing internal iterations, to reach convergence in each time step, Jameson (1991). For the present problem, a typical number of inner iterations of 100 was necessary for the convergence in each time step. In the present study, the artificial compressibility method, Chorin (1968), has been employed with a second-order centered spatial scheme.

\subsection{Turbulence modelling}

The URANS turbulence models used in this study are the Spalart and Allmaras (1992), the $k-\omega$ models (baseline and SST), Menter (1994), the OES $k-\varepsilon$ and the OES $k-\omega$ models, described in Braza et al. (2006), Bourguet et al. (2008). The Delayed Detached Eddy Simulation (DDES), Spalart et al. (2006), is employed by using the turbulence length scale from the OES modelling.

The association of the OES modelling in the RANS part of the DES/DDES is reported in a number of studies of the present research group, reported in a variety of test cases in the European research programs in aeronautics, DESIDER and "Advanced Turbulence Modelling for Aeronautics Applications Challenges" (ATAAC), Fu et al. (2012). The use of a turbulence length scale from OES in the DDES is reported in Haase et al. (2009), Bourguet

et al. (2008) and in Skopek et al. (2012). In the present study, the use of the above mentioned turbulence models allows assessment of their predictive capabilities in respect of the strongly detached unsteady flow in the cylinder array.

\section{Results for the static-case configuration}

Regarding the 2-D simulations, the unsteady loads on the central cylinder are presented versus time in Fig. 2 in comparison with the experimental results. It can be seen that these signals are qualitatively of a similar nature. 
Comparison of their rms values is presented in Table 1. As discussed in a next paragraph, higher-order frequencies similar to the experimental histories, appear in a more pronounced way in both cases and they are more pronounced in the case of the $k-\omega$-OES modeling. The predominant frequencies are shown in Table 2.

a)

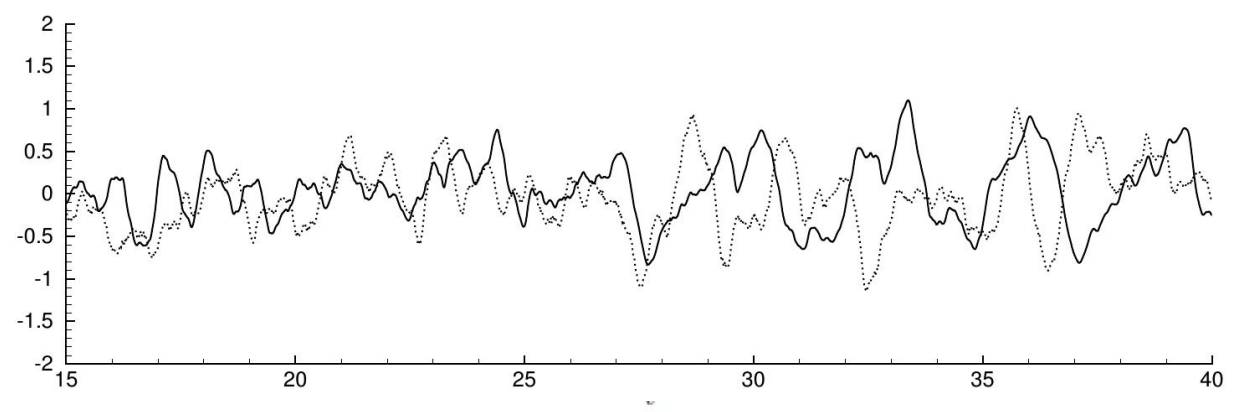

b)

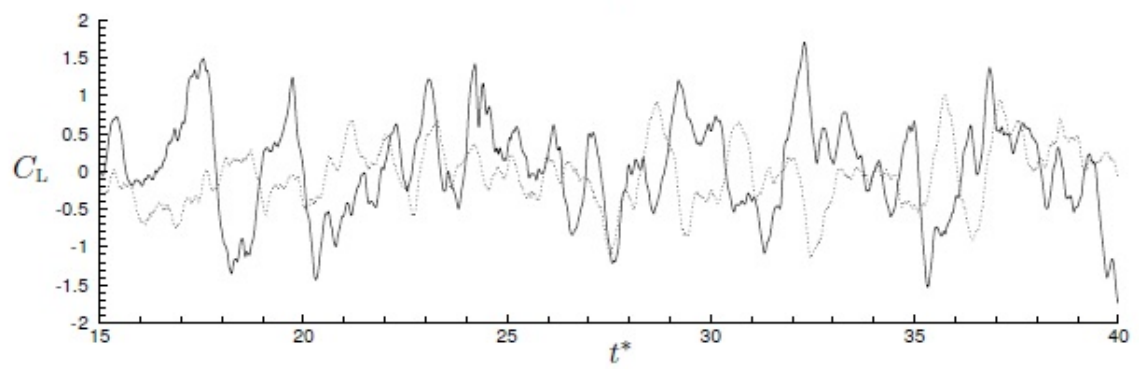

Figure 2: Comparison of the lift coefficient signals (solid line) with the DIVA experiment (dots). a) SA model; b) $k-\omega$-OES model

\begin{tabular}{|c|c|}
\hline Models & $\mathrm{C}_{L, \mathrm{RMS}}$ \\
\hline \hline SA & 0.44 \\
$k-\omega-\mathrm{SST}$ & 0.19 \\
$k-\omega-\mathrm{BL}-\mathrm{OES}$ & 0.65 \\
$k-\varepsilon-\mathrm{OES}$ & 0.46 \\
\hline \hline DIVA Experiment & 0.45 \\
\hline
\end{tabular}

Table 1: rms of the lift coefficient for different turbulence models compared with the experiment.

The $k-\varepsilon$-OES and the URANS-SA models provide the closest predictions of $r m s$ to the experiment. To our knowledge, it is the first time that 
unsteady numerical simulations through statistical turbulence modelling are able to produce a significant part of the flow physics in the cylinder bundle configuration at an inter-tube Reynolds number of 60,000, even under the two-dimensional approximation. This is an interesting issue for pre-design needs in the industrial context of nuclear reactors, as pointed out by the CEA, EDF and AREVA in the collaborative French ANR 'BARESAFE' research project in which the present study has been carried out. The $k-\omega$-SST model produces a rather high level of eddy-viscosity in the present case, having the tendency to dampen the amplitudes of the von Kármán instability and therefore to lower the rms of the lift coefficient. On the other hand, the $k-\omega$-BL-OES model has the tendency to decrease the eddy-viscosity and therefore to enhance the amplification of this instability and of the overall flow fluctuations, yielding a higher rms value. Moreover, the differences between the modeling approaches are mainly due to the fact that the flow physics of the boundary layer upstream of the first cylinder separation point correspond to laminar flow, due to the subcritical Reynolds number. The turbulence models generally assume a turbulent boundary layer, except for those models which specifically include transition modelling. Using transition modelling in the context of the present URANS approaches is a recommended issue which may improve the results. The OES approach, as shown in Jin and Braza (1994) and Bourdet et al. (2007), allows a better capturing of the boundary layer physics than standard URANS, thanks to the reduction of an excessive turbulent kinetic energy production upstream of the body, often occurring in URANS modeling when employing the Boussinesq approximation in the stress-strain law. Therefore, through this inherent reduction in the turbulence level, the laminar flow regions are dynamically captured by this approach. As an example, the well-known laminar separation bubble developed in the leading - edge region of an aerofoil at incidence and in the subcritical Reynolds number range can be captured by the present approach, as shown by Hoarau (2002) and Bourdet et al. (2007). In the present study, both SA and $k-\omega$-OES models provide close results with the experiments, although the first model produces a less rich statistical content in respect of the fluctuation amplitudes. The PSD, (Power Spectral Density) figures are presented in Fig. 3.

Three predominant frequencies appear in the spectra of the numerical study. These spectra correspond to FFT of the lift coefficient signals. The sampling rate is equal to the time step, $\Delta t=10^{-3}$ and the signals length contains an order of 50 vortex shedding periods. 


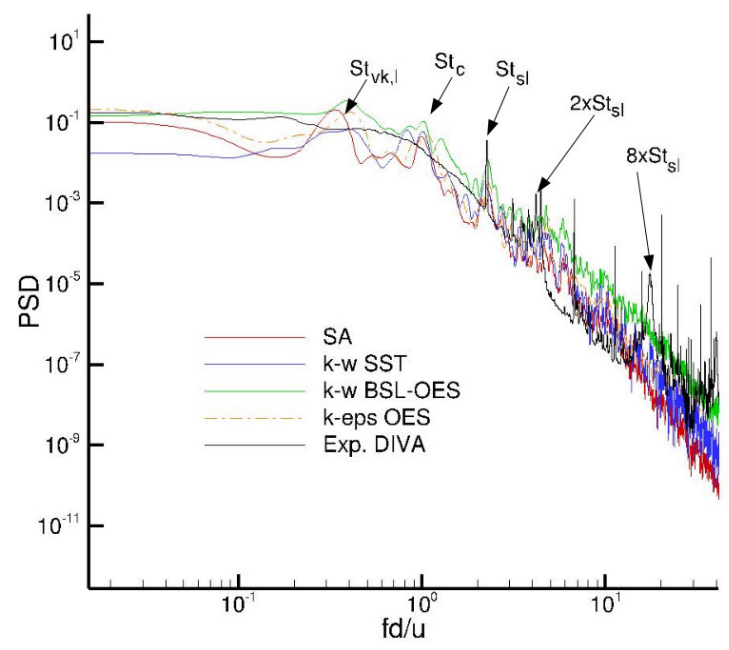

Figure 3: Power spectrum density of the lift coefficient, two-dimensional simulations using the turbulence models: Spalart-Allmaras, $k-\omega$-SST, $k-\omega$-Baseline-OES and $k-$ $\varepsilon-\mathrm{OES}$, in comparison with the experiments

The von Kármán vortex shedding dimensionless frequency is designated by $\mathrm{St}_{\mathrm{VK}}$. The Strouhal number corresponding to the accelerated (due to the confinement) inter-tube velocity is represented by $\mathrm{St}_{\mathrm{C}}$. The shear layer vortices Strouhal number is designated by $\mathrm{St}_{\mathrm{SL}}$. The experimental spectra are more 'noisy' than the numerical ones in respect of chaotic turbulence dynamics, which dampen the area of the von Kármán mode. They display predominent peaks in the area of the shear-layer instability frequency, identified from the simulations fields, as discussed in a next paragraph.

Furthermore, the Strouhal number can be also defined as a function of the inter-tube velocity, which we denote as

$$
\mathrm{St}_{., \mathrm{it}}=\frac{f d}{u_{\mathrm{it}}}=\left(\frac{P^{*}-1}{P^{*}}\right) \text { St } \quad \text { with } \quad P^{*}=\frac{P}{d},
$$

where $P^{*}$ is the reduced step and $P$ as the dimensional distance between the cylinders (pitch). The values of these predominant frequencies are summarized in Tab. 2.

The inter-tube Strouhal number corresponding to the von Kármán mode is equal to 0.34 , close to the well known value in the literature for a single cylinder in the high Reynolds number range of $60,000\left(\mathrm{St}_{\mathrm{cyl}} \approx 0.2\right.$ for a single 


\begin{tabular}{|c||c|c||c|c|}
\hline Models & St $_{\text {VK }}$ & St $_{\mathrm{VK}, \text { it }}$ & $\mathrm{St}_{\mathrm{C}}$ & $\mathrm{St}_{\mathrm{SL}}$ \\
\hline SA & 0.34 & 0.11 & 0.99 & 2.25 \\
$k-\omega-\mathrm{SST}$ & 0.36 & 0.12 & 0.99 & 2.25 \\
$k-\omega-\mathrm{OES}$ & 0.39 & 0.13 & 1.01 & 2.25 \\
$k-\varepsilon-\mathrm{OES}$ & 0.39 & 0.13 & 1.01 & 2.25 \\
\hline
\end{tabular}

Table 2: Values of predominant frequencies

cylinder). The four models practically predict the same values for the three predominant frequencies. The spectra of the experimental study display a 'bump' area in the region of the frequency peak of 0.34 . This difference is because the turbulence effects are more predominant in the experiment and lead to a more chaotic vortex shedding.

The study of the separation point cartography is presented in Fig. 4. The central cylinder is considered. The time-dependent evolution of the separation points at the cylinder's wall is illustrated. The positions on the wall are marked as a function of the angle $\alpha$ defined in Fig. 4. The black regions correspond to separation areas on the wall, as a function of time.

A periodic appearance of patterns is noticed. These patterns are associated with the instantaneous vorticity fields and illustrate the periodic behaviors depicted in the spectra concerning the predominant frequencies found in the spectra (Fig. 3).
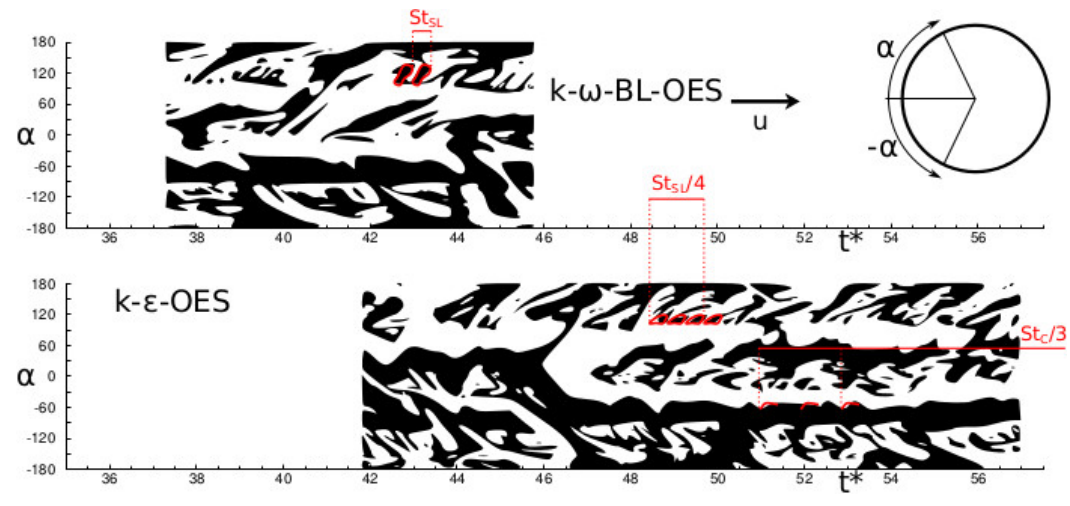

Figure 4: Separation areas versus time. The 'black' areas correspond to $\omega_{z}=0$.

The von Kármán vortex shedding, whose frequency is rather low (relatively to the two other), occurs at the rear of the cylinder, in the area 

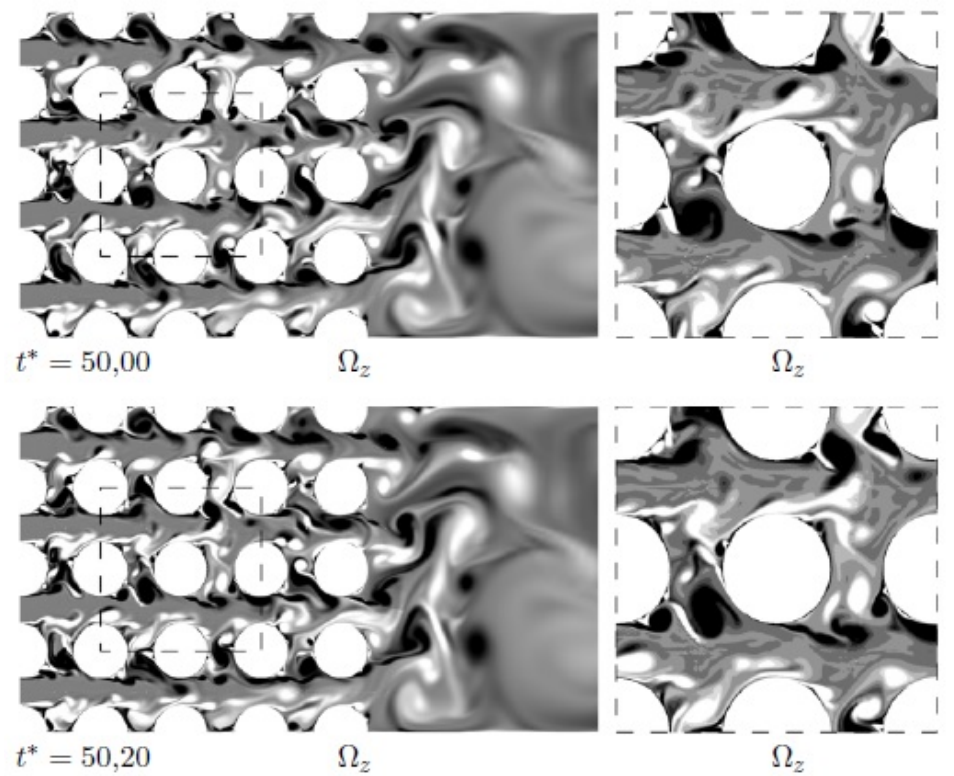

$t^{*}=50,20$

$\Omega_{z}$

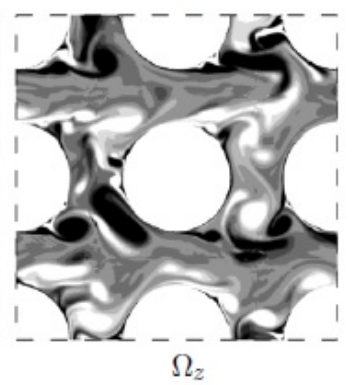

$t^{*}=50,40$

$\Omega_{2}$

$\Omega_{z}$
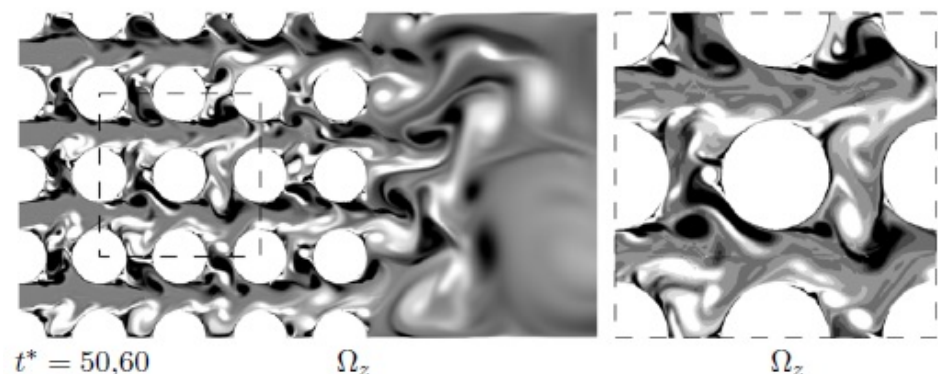

Figure 5: Instantaneous vorticity contours showing the dynamics of the shear layers and of the vortex structures in the $(x, y)$ plane with the 2-D $k-\omega$ - OES model; on the right, zoom around a central cylinder indicating the separation structure as well as the dynamics of the shear layer and of the von Kármán vortices. 

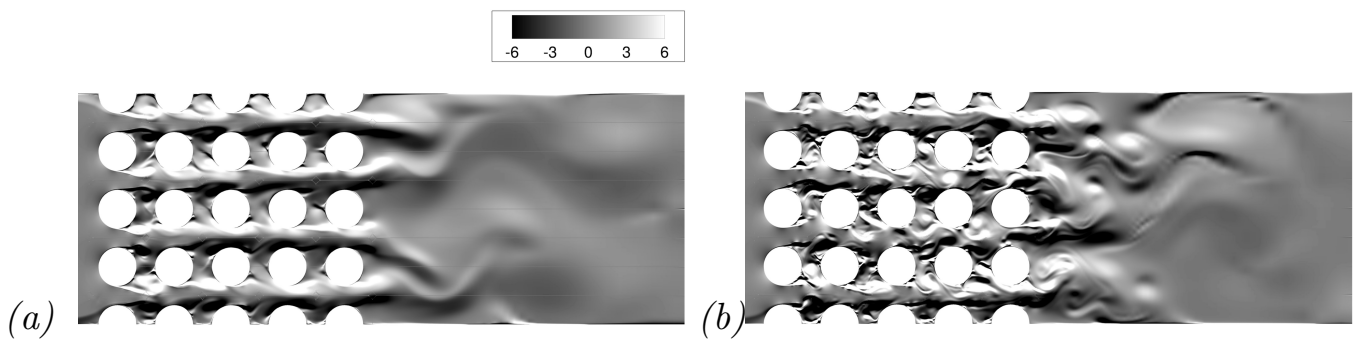

Figure 6: Instantaneous spanwise vorticity for $(a)$ Spalart-Allmaras model and $(b)$ the $k-\omega-\mathrm{BL}-\mathrm{OES}$ model

$|\alpha|>150^{\circ}$. A frequency corresponding to the shear-layer vortices is also depicted, by tracking these vortices in space-time and by evaluating their convection velocity which allowed identifying their frequency peak in the spectra. The frequency of the shear-layer eddies is higher (order 2.5), than the von Kármán vortex shedding. It is worthwhile mentioning that the majority of URANS approaches has the tendency to dampen the instability related to the appearance of the shear-layer mode, as a consequence of an excessive kinetic energy production upstream of the first cylinder. As previously stated, this is due to an excessive level of turbulent viscosity, usually produced by the Boussinesq law and by the standard values of the modeling constants in URANS. This excessive turbulence viscosity has the tendency to dampen the instability onset. A good representation of the shear-layer instability is achieved in the present study. This fact plays an important role in the evaluation of the amplitudes of the fluid forces. Finally, the third frequency is linked to a phenomenon related to the impact of coherent structures on the zone $|\alpha| \approx 60^{\circ}$. This is the area where the structures issued by the upstream rows of cylinders are destroyed. This predominant frequency is linked to the confinement, caused by the adjacent cylinders in the bundle.

The present study associates the appearance of these frequency modes with the related instabilities and vortex structures, Fig. 4 and 5, where these frequencies are linked to the physical phenomena of the von Kármán and shear-layer instability vortices. Moreover, the vorticity iso-contours corresponding to OES reveal a more rich ensemble of vortex structures in the flow than in the other URANS approaches (fig. 6) and the energy of the corresponding frequency peaks is higher in the spectra. These peaks as well as the organized vortex structures are directly linked with the unsteady load am- 


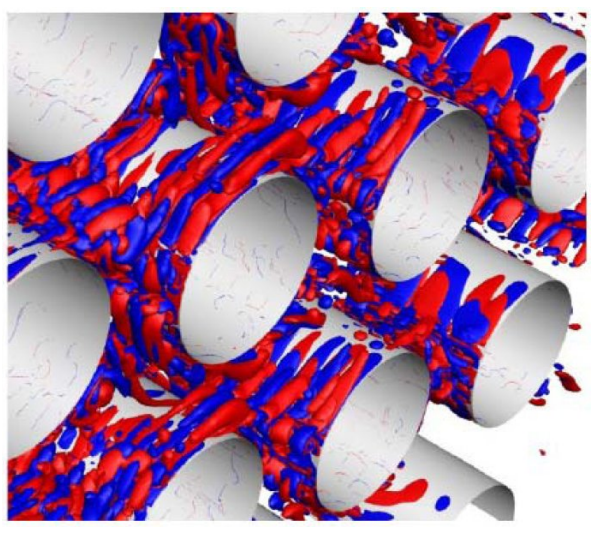

(a)

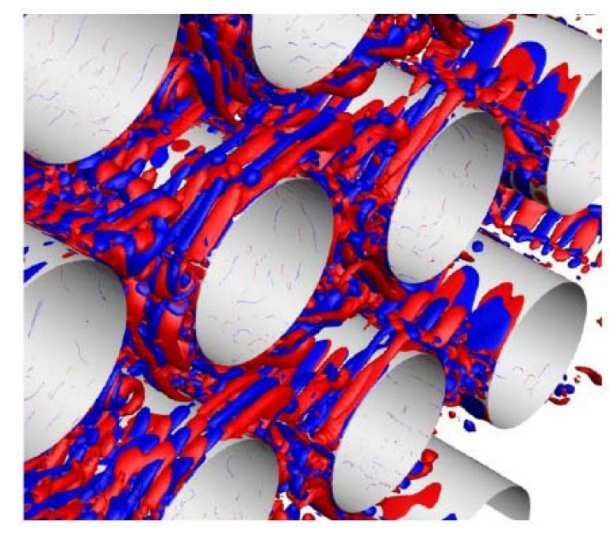

(b)

Figure 7: $\omega_{z}$ vorticity iso-surfaces for the DDES- $k-\omega$-OES model $(b)$ within a time interval of 10 dimensionless time-units.

plitudes which are important in the dynamics of the fluid-elastic instability, studied in section 4 .

Fig. 7 shows the onset of the three-dimensional motion by means of streamwise and $\omega_{z}$ vorticity within a time interval of $10 \mathrm{~s}$. The initial conditions are those of flow at rest. The DDES $-k-\omega$-OES modelling has been used. The onset of three-dimensional motion is organized according to counter-rotating cells of positive-negative vorticity, displaying a regular predominant wavelength, $\lambda=0.2 D$. This structure persists as a function of time, as shown within the time interval of $10 \mathrm{~s}$ in the present simulations. This flow pattern is in qualitative accordance with experimental studies by $\mathrm{Wu}$ et al (2005), where the cross-sectional size of secondary vortices range between $0.2 \mathrm{D}$ and $0.5 \mathrm{D}$ past a normal flat plate in the Reynolds number range 1800-27,000. Moreover, this wavelength is comparable to other simulations on a single cylinder at high Reynolds number Bourguet et al. (2008), El Akoury et al. (2009).

\section{Simulation of the fluid-structure interaction in the case of the central cylinder one-DOF motion}

In this section the central cylinder is allowed to move freely in the vertical direction. A first part of this numerical study is 2-D by using in the CFD part the $k-\omega$-BL-OES model, studied in section 3. A second part of the study is 3 -D by using the DDES- $k-\omega$-BL-OES model, after the static-case study presented in section 3 . The Reynolds number is of 60,000 inter-tube. With regard to the solid structure, three important parameters for the study of the fluid-elastic instability are: the mass of the cylinder per unit length $m$, 
the natural frequency $f_{s}$ of the solid structure and the damping ratio $\zeta$. The definitions of these variables vary widely. These variables are often defined in respect of the fluid medium at rest. In the present study, these parameters are defined in vacuum (practically in air). All the structural parameters and their definitions are listed in Table 3.

The equation of motion of the central cylinder has been non-dimensional by using the flow parameters above and is given by Equation 2 .

$$
m^{*} \ddot{y}^{*}+b^{*} \dot{y}^{*}+k^{*} y^{*}=c_{y}\left(t^{*}\right)
$$

In this equation, $y$ is the displacement of the cylinder in the vertical axis, perpendicular to the cross flow and $c_{y}$ is the unsteady, non-dimensional lift force per unit length in the $y$ axis.

\begin{tabular}{|l|c|l|}
\hline Variable name & Definition & Description \\
\hline reduced velocity $\left(U^{*}\right)$ & $\frac{U}{f_{s} D}$ & $U$ is inflow velocity, $f_{s}$ is structure frequency \\
mass $\left(m^{*}\right)$ & $\frac{m}{\frac{1}{2} \rho_{f} D^{2} L}$ & $m^{*}=6.76$ is fixed, L - cylinder length \\
damping $\left(b^{*}\right)$ & $\frac{b}{\frac{1}{2} \rho_{f} D U L}$ & $b^{*}=\frac{4 S c}{U^{*}}$ \\
Scruton number $(\mathrm{Sc})$ & $\frac{\pi \zeta m^{*}}{\text { or } \frac{m \delta}{\rho D^{2}}, \text { with } \delta=\frac{2 \pi \zeta}{\sqrt{1-\zeta^{2}}}}$ \\
stiffness $\left(k^{*}\right)$ & $\frac{k}{\frac{1}{2} \rho_{f} U^{2} L}$ & $k^{*}=\left(\frac{2 \pi}{U^{*}}\right)^{2} m^{*}$ \\
\hline damping ratio $(\zeta)$ & $\frac{b^{*}}{2 \sqrt{m^{*} k^{*}}}$ & changed using $m^{*}$ and Sc \\
reduced velocity $\left(U^{*}\right)$ & $2 \pi \sqrt{\frac{m^{*}}{k^{*}}}$ & $f_{s}^{*}=\frac{1}{U^{*}}$ \\
\hline
\end{tabular}

Table 3: Variable definitions

\subsection{2-D simulation results}

The influence of the time step on the induced cylinder's movement is firstly presented. The study has been carried out by means of the $k-\omega$-BLOES model and for a Reynolds number of 60,000 . The results are shown in Fig. 8 .

A good convergence of the results is obtained for the time-steps 0.01 and 0.02. The time-step $\Delta t=0.005$ indicates a slight difference in the amplitudes 


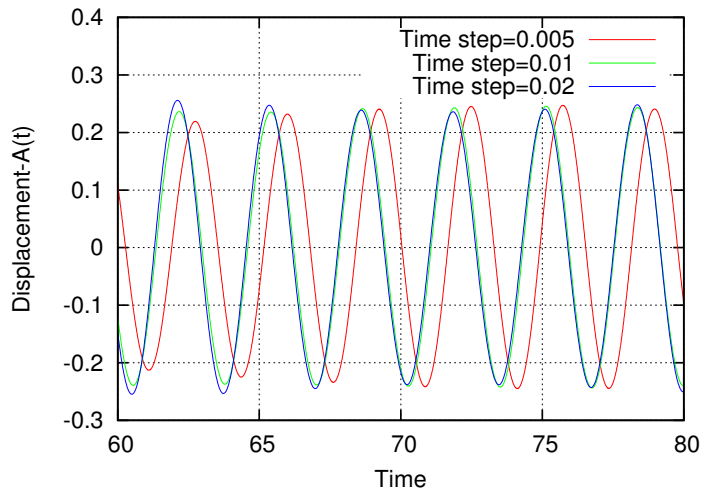

(a)

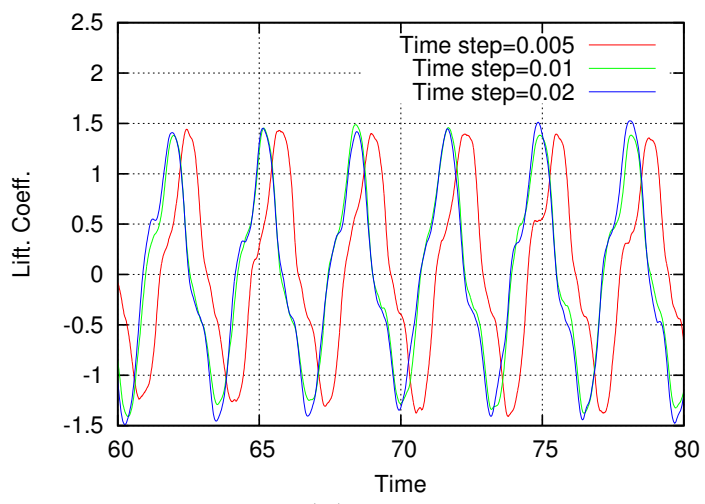

(c)

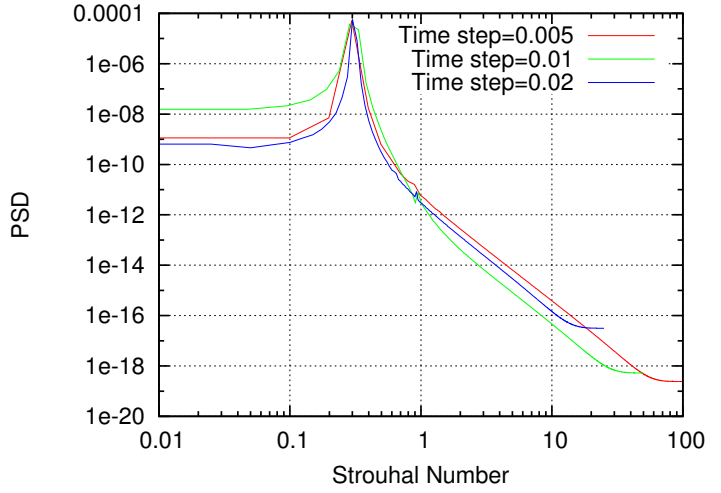

(b)

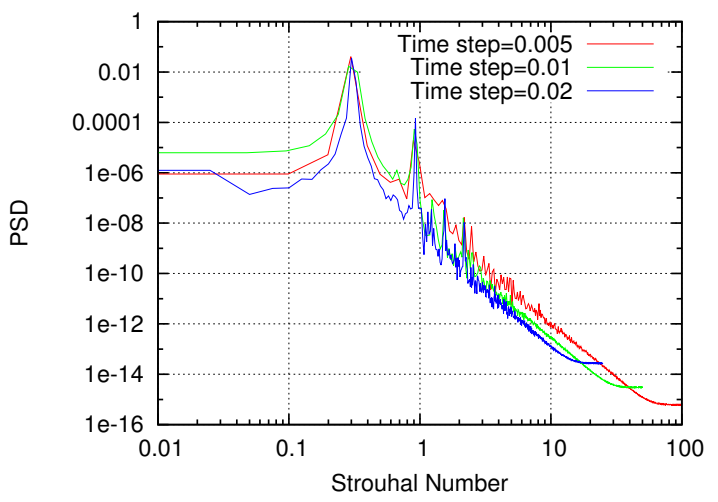

(d)

Figure 8: Time evolution of the displacement (a) and the lift coefficient (c) and spectra of the displacement (b) and the lift coefficient (d) for different time steps. 


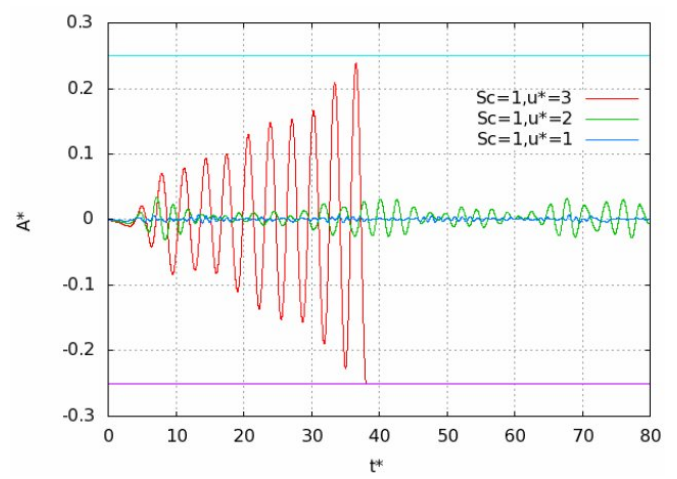

(a)

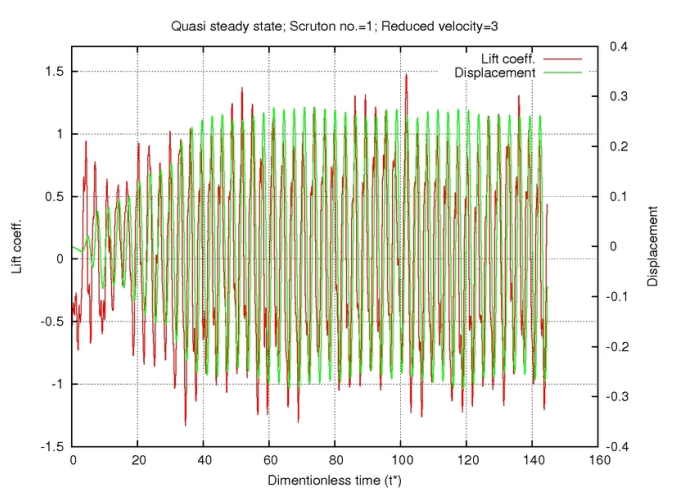

(b)

Figure 9: (a) Cylinder displacement for $\mathrm{Sc}=1$ and different reduced velocities, (b) Quasisteady state for $\mathrm{Sc}=1$ and $u^{*}=3$

and in the phase. Reducing the time step beyond a 'limit' value in respect of the grid refinement, which remains the same, typically leads to a loss of convergence. This is the case in the present results corresponding to the finer time-step. A finer grid would be necessary in order to respect the decrease of the truncation error obtained by the time-step reduction. The intermediate time-step value is adopted therefore $(\Delta t=0.01)$, in order to provide realizable computations in the 3-D dynamic case.

The onset of the fluid-elastic instability in MIV (Movement Induced Vibration) is shown in Fig. 9(a). This phenomenon is characterized by the negative damping of the displacement amplification. This occurs because the energy supplied by the fluid cannot be instantaneously destroyed by the solid structure, which is excited by a progressively increasing energetic level. This energy does not have the time to be consumed and consequently creates a negative damping appearance. According to the Connors (Connors (1970)) diagram and to the studies by Paidoussis and Price (1988), as well as Granger and Paidoussis (1996), this instability particularly occurs for values of the Scruton number and of reduced velocity beyond $(1,4)$. For lower values of these parameters, the displacement amplitude versus time is either dampened or reaches a steady state, Fig. (9).

For values of the reduced velocity up to 3 , the frequency of the displacement and of the lift coefficient is the same as the fluid's vortex shedding frequency (Fig. 8 (b) and (d)). This corresponds to a lock-in behavior 
characterizing the VIV mechanism. An important feature occurring as the reduced velocity increases, is the increase of the phase lag in the displacement (Fig. 10 and 11) for reduced velocities included in the MIV range, whereas there is no phase-lag for reduced velocities lower or equal to 3 (Fig. 9(b)).

Figure 10 and 11 shows the phase-lag development between lift and displacement of the central cylinder for two values of the Scruton number and for various reduced velocities. The two Scruton numbers selected correspond in the literature to regimes before and after the Movement Induced Vibration. For $\mathrm{Sc}=1$, the phase-lag is found to increase in the range of reduced velocities from 5 to 14 . For $\mathrm{Sc}=5$, the increase of the phase-lag is less drastic. The phase-lag values are presented as a function of the reduced velocity in Table 4. In all cases, the increase of the reduced velocity displays the appearance of higher frequencies in the lift fluctuation. These frequencies do not appear in the body's oscillation.

The reduced velocity $\left(U^{*}\right)$ in the simulations is increased by decreasing the solid structure's natural frequency $f_{s}$. Figure $12($ a) shows the vorticity plot, where the central cylinder becomes unstable at $\mathrm{Sc}=1, U^{*}=3$. Figure $12(\mathrm{~b})$ shows the rms of the cylinder's displacement as a function of the reduced velocity for three values of Sc. The low value of the mass-damping parameter $(\mathrm{Sc}=0.0127$ corresponding to the red curve) shows a sharp increase in the amplitude of the oscillations at low reduced velocities. The instability seems to occur at $U^{*} \approx 1.2$. A further increase in the value of the Scruton $\mathrm{Sc}=1$ delays the occurrence of this instability. The curve shows a sudden increase in the oscillations for the reduced velocity $U^{*}=3$. The green curve in Figure 12(b) shows the response of the cylinder for the mass-damping ratio $\mathrm{Sc}=5$. Table 4 summarizes the global parameters, the Strouhal number of the lift coefficient, of the cylinder's displacement and the phase-shift angle (rad) between the lift and the cylinder's position, as a function of the reduced velocity for the case of Scruton number 1. It is shown that the phase-lag is practically equal to zero for the low values of the reduced velocities (VIV regime) and it increases progressively in the range of the higher values (MIV regime).

Figure 12(c) and (d) shows the drag and lift coefficient variations for the three cases by increasing the reduced velocity.

The static case simulation shows the Strouhal number of the order $S t=0.33$, a purple line in Figure 13(a). It is interesting to show the variation of this response frequency with the change in the Scruton number (Sc). In the case of the low value of the mass-damping parameter $\mathrm{Sc}=0.0127$, the fluid- 


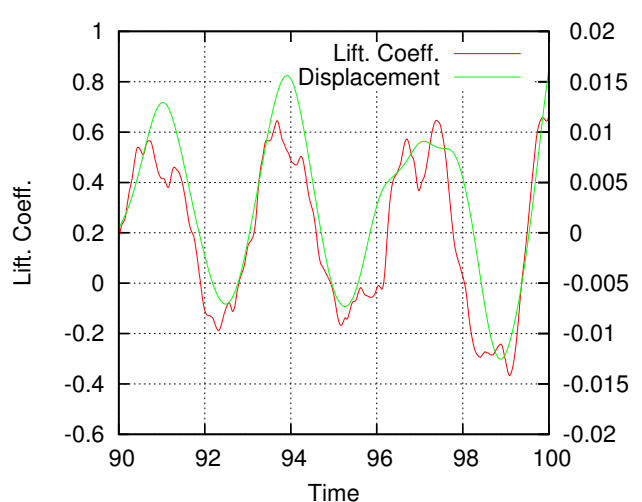

(a)

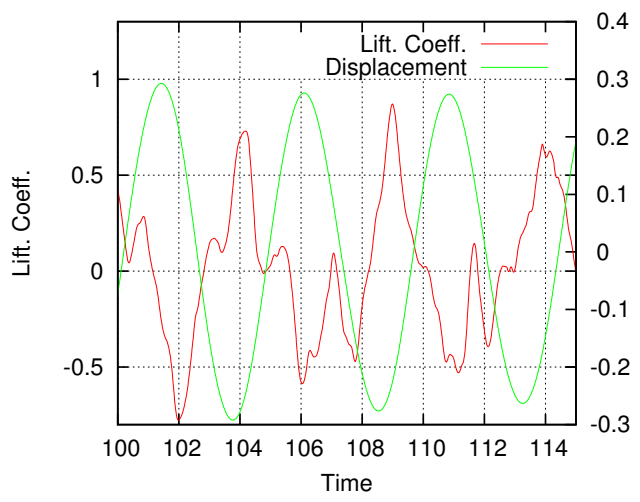

(c)

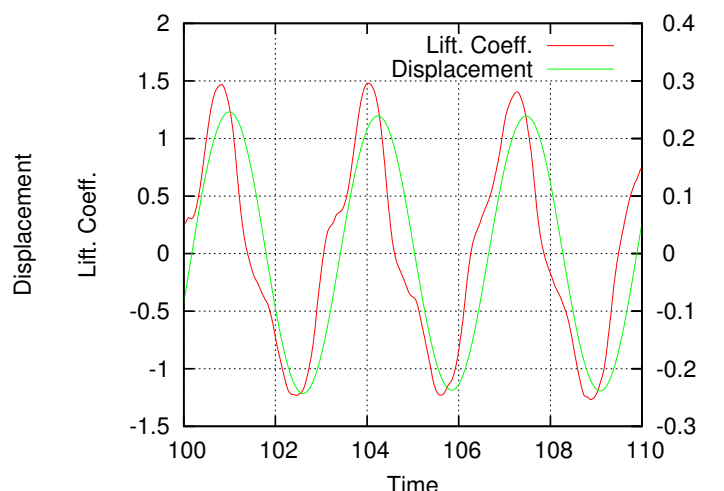

(b)

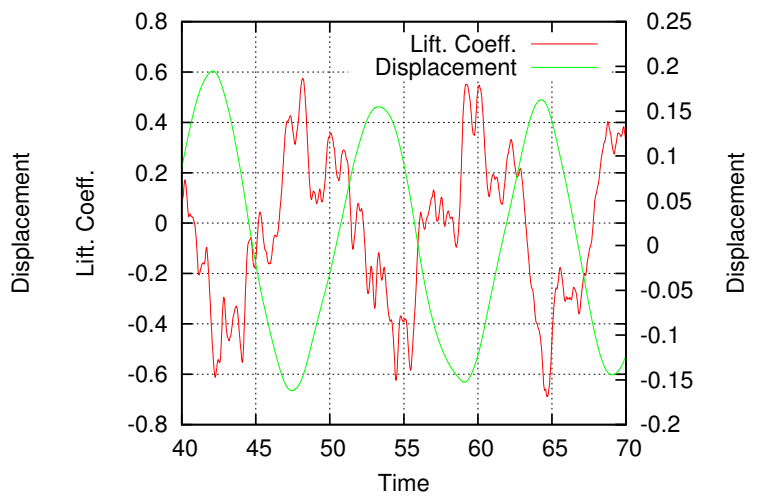

(d)

Figure 10: Time-evolution of the displacement and the lift coefficient for $\left(\mathrm{Sc}=1, u^{*}=2\right.$ (a), $\mathrm{Sc}=1, u^{*}=3$ (b), $\mathrm{Sc}=1, u^{*}=5$ (c), $\mathrm{Sc}=1, u^{*}=10$ (d). 


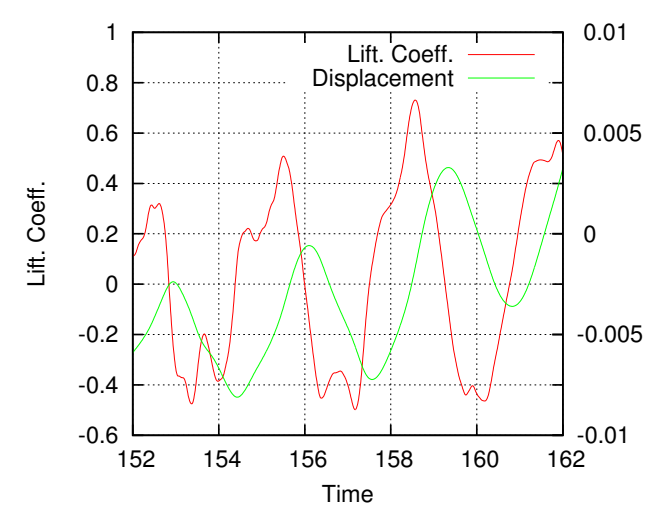

(a)

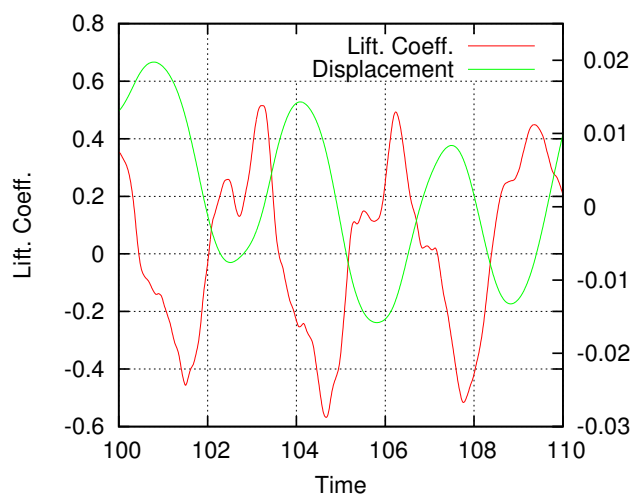

(c)



(b)

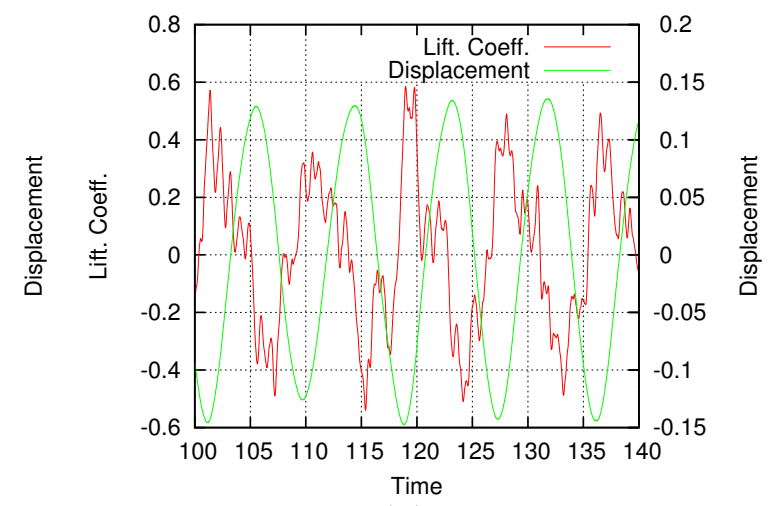

(d)

Figure 11: Time-evolution of the displacement and the lift coefficient for $\mathrm{Sc}=5, u^{*}=2$ (a), $\mathrm{Sc}=5, u^{*}=3$ (b), $\mathrm{Sc}=5, u^{*}=5$ (c), $\mathrm{Sc}=5, u^{*}=10$ (d). 


\begin{tabular}{|c|cc|cc|cc|}
\hline$u^{*}$ & $\left(\mathrm{St}_{C_{L}}\right)$ & $\left(\mathrm{St}_{A^{*}}\right)$ & $C_{D, \mathrm{rms}}$ & $C_{L, \mathrm{rms}}$ & $\operatorname{Shift}\left(A_{\mathrm{rms}}^{*}\right)$ & $A_{\max } * D$ \\
\hline 1 & 0.351 & 0.351 & 0.412 & 0.289 & $0.00 \pi$ & 0.007 \\
2 & 0.384 & 0.384 & 0.394 & 0.374 & $0.02 \pi$ & 0.038 \\
3 & 0.331 & 0.331 & 1.096 & 0.590 & $(1+0.07) \pi$ & 0.277 \\
4 & 0.261 & 0.261 & 0.603 & 0.321 & $(1+0.074) \pi$ & 0.316 \\
5 & 0.228 & 0.228 & 0.527 & 0.273 & $(1+0.078) \pi$ & 0.292 \\
6 & 0.192 & 0.192 & 0.449 & 0.222 & $(1+0.08) \pi$ & 0.258 \\
7 & 0.175 & 0.175 & 0.470 & 0.254 & $(1+0.09) \pi$ & 0.278 \\
8 & 0.159 & 0.159 & 0.500 & 0.339 & $(1+0.098) \pi$ & 0.311 \\
10 & 0.101 & 0.101 & 0.290 & 0.197 & $(1+0.104) \pi$ & 0.194 \\
12 & 0.098 & 0.098 & 0.336 & 0.240 & $(1+0.109) \pi$ & 0.238 \\
14 & 0.113 & 0.113 & 0.362 & 0.300 & $(1+0.11) \pi$ & 0.276 \\
16 & 0.110 & 0.110 & 0.370 & 0.330 & $(1+0.117) \pi$ & 0.334 \\
18 & 0.106 & 0.106 & 0.430 & 0.399 & $(1+0.119) \pi$ & 0.365 \\
20 & 0.101 & 0.101 & 0.421 & 0.400 & $(1+0.122) \pi$ & 0.387 \\
\hline
\end{tabular}

Table 4: Summary of the global parameters and of the phase-lag between the cylinder displacement and the forces (in red) versus the reduced velocity for the dynamic case with Scruton number of 1 and mass ratio of 6.76 .

elastic instability (high amplitude oscillations) occurs without a decrease in the Stouhal number below 0.33. Figure 13(b) shows the response frequency spectra for the Scruton number $\mathrm{Sc}=0.0127$ for different values of the reduced velocity. The red curve for the Scruton Sc1 shows a smooth transition and follows the structure's natural frequency curve. This feature also is reflected in Figure 13(c). This figure shows the spectra for the response of the cylinder at $\mathrm{Sc}=1$ for reduced velocities below and above the critical value $U^{*}=3$. Furthermore, the increase in the mass-damping ratio, enhances this transition.

The spectra of the cylinder's displacements at $\mathrm{Sc}=5$ and reduced velocities $U^{*}=3,6,7,10$ are shown in Figure $13(\mathrm{~d})$. The spectrum at the reduced 


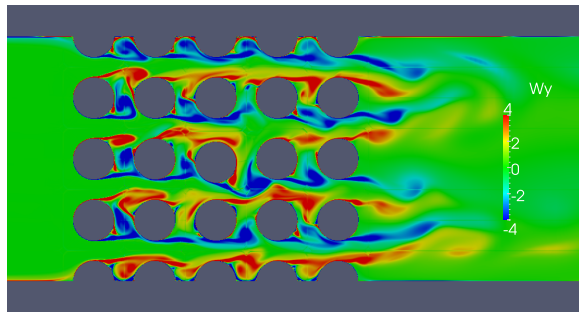

(a)

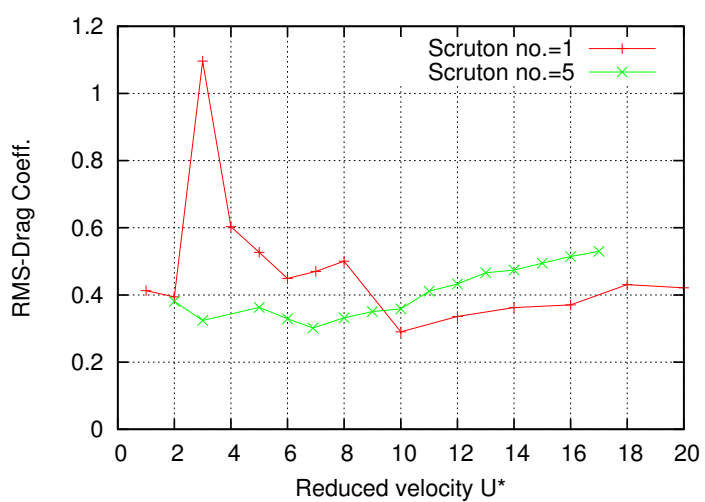

(c)

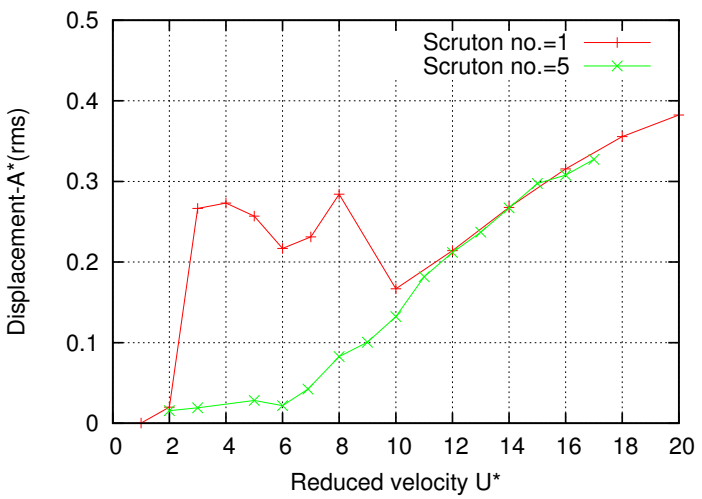

(b)

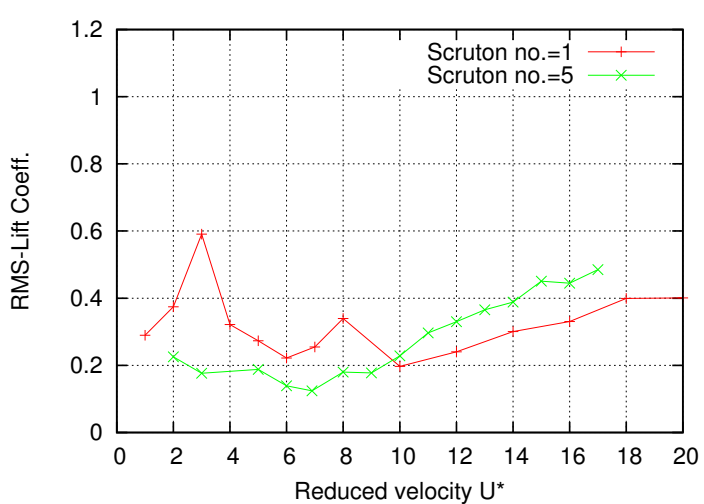

(d)

Figure 12: 1-DOF response of the central cylinder for $S c=0.0127,1$ and 5, (a) Vorticity plot for $S c=1, U^{*}=3$, (b) Root mean square non-dimensional displacement Vs reduced velocity $\left(U^{*}\right)$, (c) Drag coefficient variation with increasing reduced velocity (d) Lift coefficient profile for increasing reduced velocity

velocity $U^{*}=3$ shows a peak about 0.37 , while the spectra for $U^{*}=6,7$ indicates peaks at 0.12 and at about 0.37 . The spectrum at the reduced velocity $U^{*}=10$ shows a peak at the dimensionless frequency of 0.12 .

Figure 14 shows a comparison of the cylinder's response spectra between the experiment and the simulations. A good agreement is shown concerning the predominent frequency close to the Strouhal number of 0.33, which corresponds to the fluid's vortex shedding frequency, as expected in the context of the VIV mechanism, in the low reduced velocity range. 


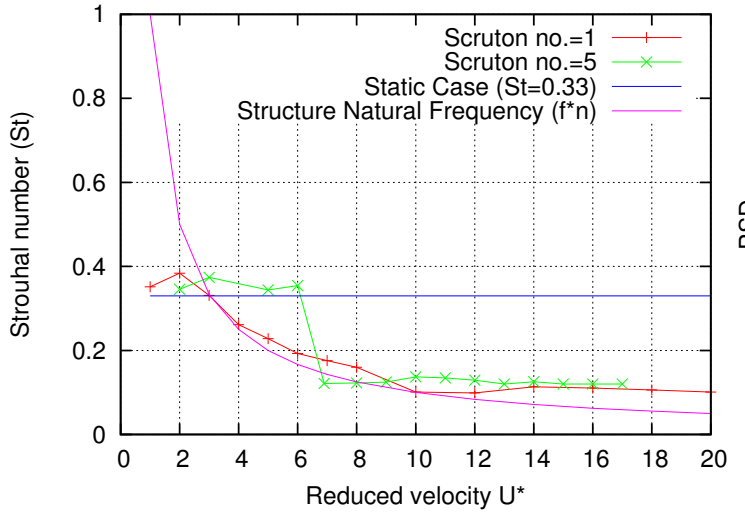

(a)

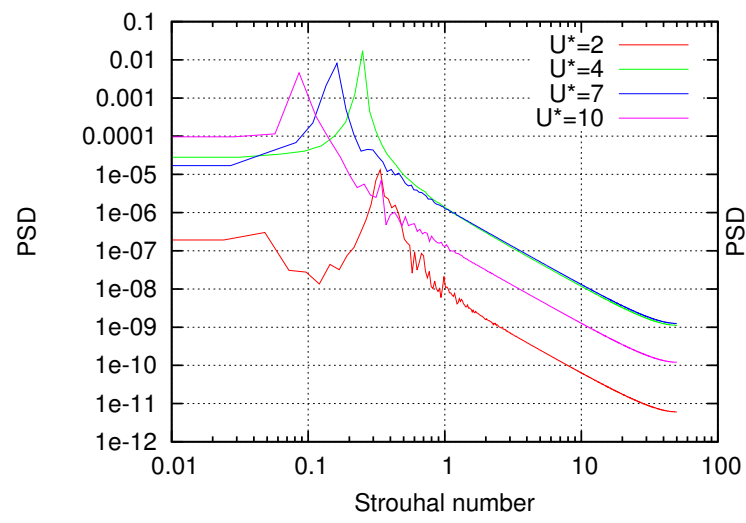

(c)

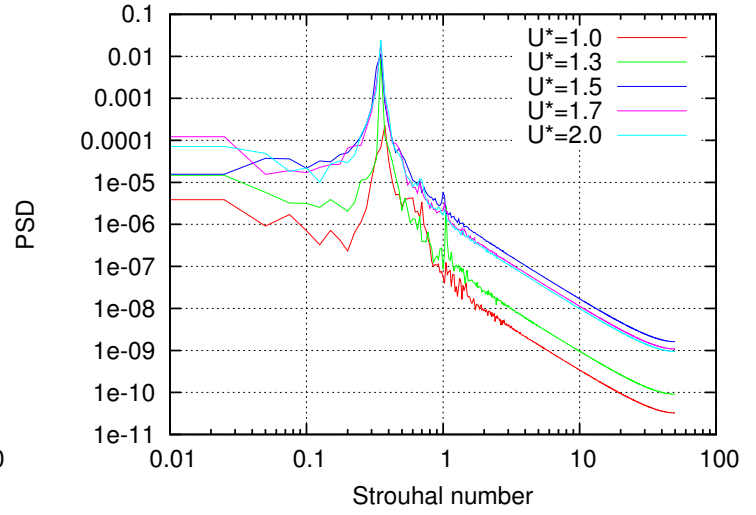

(b)

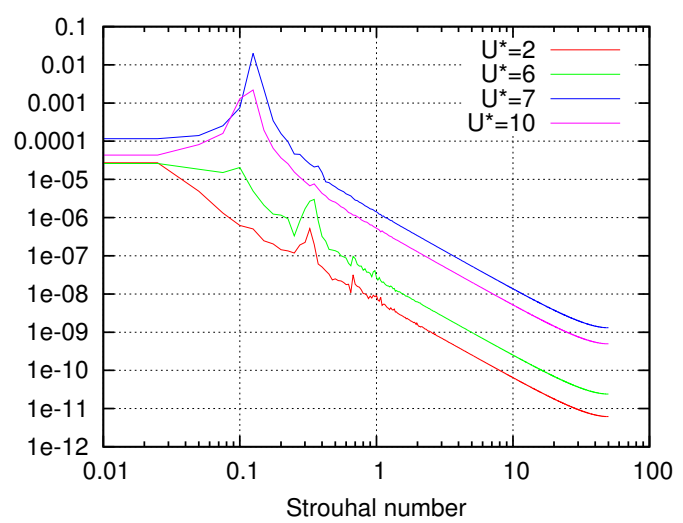

$(\mathrm{d})$

Figure 13: 1-DOF response of the central cylinder for different values of Scruton number $(S c)$ and reduced velocity $\left(U^{*}\right)$ (a) Variation of Strouhal number $(S t)$ in different cases; (b) Frequency spectra of the central cylinder displacement for $S c=0.0127$; (c) Frequency spectra of the cylinder displacement for Scruton number $S c=1$; (d) Frequency spectra of the displacement for Scruton number $S c=5$

\subsection{2-D vs 3-D comparison}

The turbulent motion is inherently three-dimensional, therefore it is interesting to examine the influence of the third dimension on the results at high Reynolds numbers. Static case simulations carried out by LES at low upstream Reynolds number $R e_{p}=2840$, (Shinde et al. (2013)), indicated that the flow is nearly invariant in the third $(z)$ direction, except for a weak large-scale spanwise wavelength undulation. However, in the higher Reynolds 


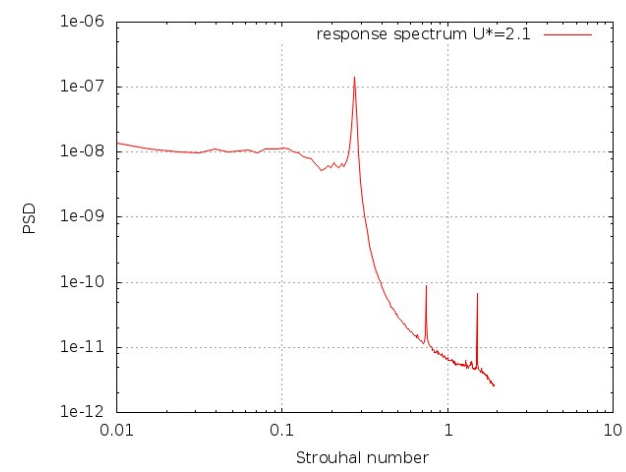

(a)

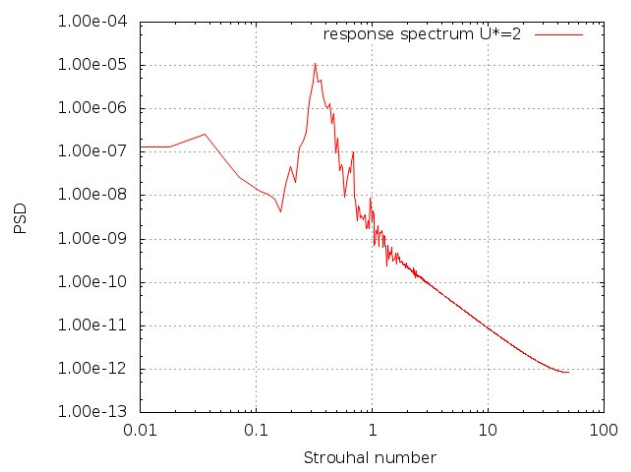

(b)

Figure 14: Comparison of the dynamic response PSD between the experiment and the 2-D simulations, $k-\omega$-BL-OES model.

number range, the three-dimensional effects are more significant.

Figure 15 compares the response of the central cylinder in 2-D and 3$\mathrm{D}$ simulations. The computational domain length in spanwise direction is 1D. Figure 15(a) and Figure 15(b) are time-histories of the cylinder's displacement and of the lift coefficient of the central cylinder respectively. The simulations have been carried out for the unstable case, at $\mathrm{Sc}=1$ and $U^{*}=3$. The 3-D simulation shows about $30 \%$ increase in the amplitude of the oscillations than in the two-dimensional case, (red curve in Figure 15(a)). The lift coefficient versus time shows irregular fluctuations in 3-D compared to the 2-D signal (see Figure 15(b)). Figures 15(c) and (d) show the spectra in 2-D and 3-D for the cylinder's displacement and the lift coefficient respectively. The spectra of the response signal of the cylinder show the same frequency of the oscillations in 2-D and 3-D simulations. This confirms theoretical considerations of the fluid-elastic instability, as well as experimental observations reporting that the instability mainly appears in the perpendicular direction in respect of the cross flow. In addition, the lift coefficient spectrum in 3-D displays higher frequencies, indicating that the turbulence energy increases due to the spanwise direction dynamics. There are frequency peaks at Strouhal numbers $S t=0.6$ and $S t=0.9$ approximately, which are related to the first and second harmonics of the main vortex shedding. The peak at 0.6 is a first harmonic of the main vortex shedding and indicates a coupling with the drag coefficient, which is mainly influenced, as known, by this first harmonic of the Strouhal number. 


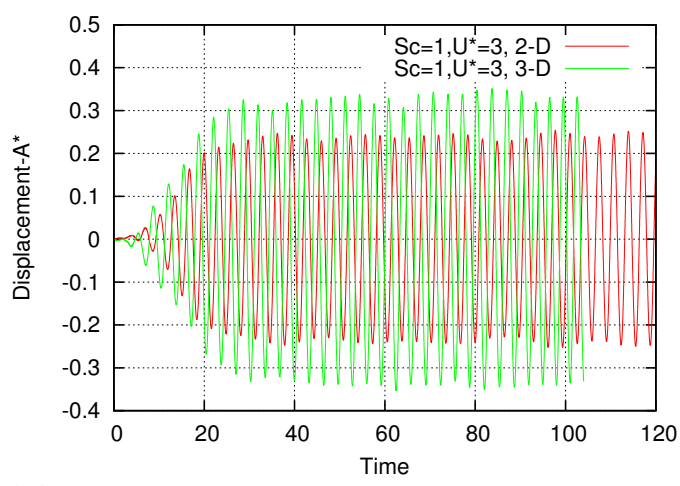

(a)

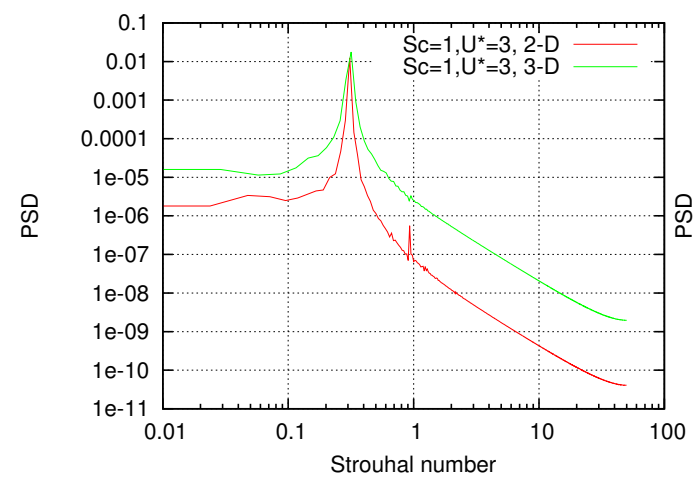

(c)

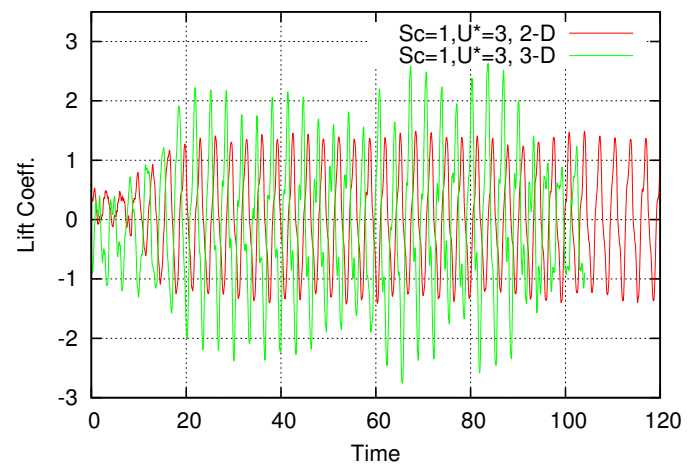

(b)

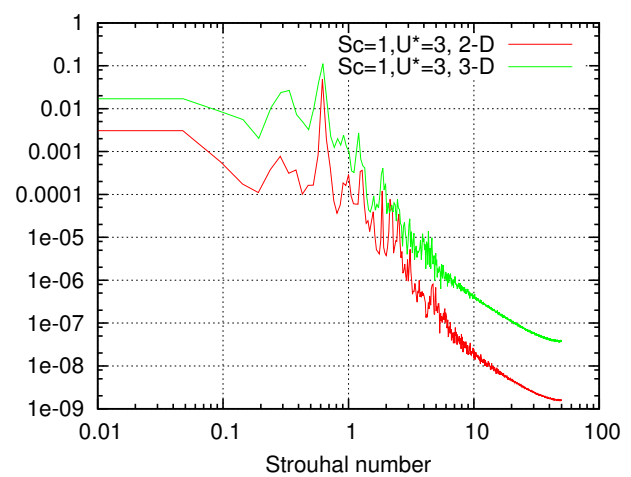

(d)

Figure 15: Comparison between the 2D simulations carried out by the $k-\omega-$ OES model and 3D simulations carried out by the DDES $-k-\omega$-OES model: (a) and (b): timehistories of the cylinder displacement and of the lift coefficient respectively. (c) and (d) spectra of the above time-histories.

\section{Conclusions}

In this paper, the numerical simulation of the fluid-elastic instability in a cylinder bundle has been carried out at in a high Reynolds number range, for various reduced velocity values and for two Scruton numbers corresponding to the low and high range of the Connors diagram. The turbulence motion effect has been captured by means of URANS and DDES modelling. The spontaneously amplified fluid-elastic instability of a central cylinder of the array, freely moving in one DOF has been produced by the present simulations and the phase-lag between the vertical oscillations displacement in respect of the forces has been assessed. It has been shown that a first stage of the 
instability corresponds to a VIV lock-in mechanism of the displacements frequency for the Scruton number 1 and reduced velocities lower or equal to 5, as well as for the Scruton number 5 and reduced velocities lower than 3 . The successive stage for higher values of reduced velocity is an MIV mechanism where the displacement frequency decreases and tends towards an asymptote, slightly higher than the natural frequency of the solid structure. The present study shows significant 3 -D effects in the amplitude variation, whereas the main frequency peaks are practically unaffected by the three-dimensionality. The two-dimensional simulations, which are much faster than the 3-D ones, produce the predominant instability frequencies but under-estimate the fluctuation amplitudes. These facts are significant for the design of the cylinder bundles in nuclear reactor engineering.

\section{Acknowledgments}

This paper has received a computing grant allocation from the three French Government computing Centers of High Education and Research, CINES, IDRIS and CALMIP, as well as computing allocation in the context of the European initiative PRACE, using the CEA's Curie supercom-

puter. This study is a collaboration between Electricité de France (EDF), Commisariat d'Energie Atomique (CEA) and AREVA in the context of the French research program BARESAFE supported by the Agence Nationale de la Recherche (ANR). Part of the methods for modelling the fluid-structure interaction is developed in the context of the national research platform RTRA-SMARTWING, funded by the French Foundation STAE, "Sciences et Technologies pour l'Aeronautique et l'Espace".

\section{References}

Barbut, G., Braza, M., Hoarau, Y., Barakos, G., Svrain, A., Vos, J.B., 2010. Prediction of transonic buffet around a wing with flap., Notes on Numerical Fluid Mechanics and Multidisciplinary Design, Volume 111 : Progress in Hybrid RANS-LES Modelling by Shia-Hui Peng, Piotr Doerffer, Werner Haase, 191-204.

Blevins, R., 1974. Fluid-elastic whirling of a tube row. J. of Pressure Vessel Tech. 96, 263-267. 
Blevins, R. D., 1979. Formulas for natural frequency and mode shapes. Krieger Publishing Company.

Bourdet, S., Braza, M., Hoarau, Y., El Akoury, R., Ashraf, A., Harran, G., Chassaing, P., Djeridi, H., 2007. Prediction and physical analysis of unsteady flows around a pitching airfoil with the dynamic mesh approah. Eur. J. Comp. Mech. 16(3/4), 451-476.

Bourguet, R., Braza, M., Harran, G., El Akoury, R., 2008. Anisotropic organised eddy simulation for the prediction of non-equilibrium turbulent flows around bodies. J. of Fluid and Struct. 24(8), 1240-1251.

Braza, M., Faghani, D., Persillon, H., 2001. Successive stages and the role of natural vortex dislocations in three-dimensional wake transition. J. of Fluid Mech. 439, 1-41.

Braza, M., Perrin, R., Hoarau, Y., 2006. Turbulence properties in the cylinder wake at high reynolds number. J. of Fluid and Struct. 22, 7551-771.

Chen, S. S., 1983. Instability mechanisms and stability criteria of a group of circular cylinders subjected to cross flow. part i: Theory. J. of Vibration, Acoustics, Stress and Reliability in Design 105(1), 51-58.

Chorin, A., 1968. Numerical solution of the navier-stokes equations. J. Math. Computation 22, 745 .

Connors, H. J., 1970. Flow induced vibration of heat exchanges. New York:ASME Fluidelastic vibrations of tube arrays excited by cross flow, $42-56$.

Deloze, T., Hoarau, Y., Dušek, J., 2010. Chimera method applied to the simulation of a freely falling cylinder in a channel. European Journal of Computational Mechanics 19, 575-590.

El Akoury, R., Braza, M., Mockett, C., Perrin, R., Reimann, T., Revell, A., Craft, T., Laurence D., Hoarau,Y., Thiele, F., 2009. The IMFT circular cylinder flow case CFD comparison. In Book from: Haase, W., Braza, M., Revel, A., Sci Eds. DESider - A European Effort on Hybrid RANSLES Modelling, Notes on Numerical Fluid Mechanics and Multidisciplinary Design, 103, Publisher Springer, 
Ferreira-Perez, S., 2013. Simulation numérique et modélisation de l'interaction fluide-structure et étude vibratoire de deux cylindres en tandem nombre de Reynolds élevé. Engineering diploma thesis, ENSEEIHTIMFT.

Fu, S., Haase, W., Peng, S. H., Schwamborn, D., 2012. Progress in Hybrid RANS-LES Modelling. Notes on Numerical Fluid Mechanics and Multidisciplinary Design 117, 59-75.

Granger, S., Paidoussis, M., 1996. An improvement to the quasi-steady model with application to cross-flow-induced vibration of tube arrays. J. Fluid Mech. (320), 163-184.

Grossi, F., Braza, M., Hoarau, Y., 2014. Prediction of Transonic Buffet by Delayed Detached-Eddy Simulation. AIAA J., in print.

Haase, W., Braza, M.,Revell, A., 2009. DESiderA European Effort on Hybrid RANS-LES Modelling. Vol. 103, Notes on Numerical Fluid Mechanics and Multidisciplinary Design, Springer.

Hoarau, Y., 2002. Analyse Physique Par Simulation Numérique et Modélisation Des Écoulements Décollés Instationnaires Autour de Surfaces Portantes. Thèse de doctorat, INPT (Institut National Polytechnique de Toulouse).

Jameson, A., 1991. Time dependant calculations using multigrid, with application to unsteady flows past airfoils and wings. AIAA paper 91-1596.

Jin, G., Braza, M., 1994. A two-equation turbulence model for unsteady separated flows around airfoils. AIAA J. 32(11), 2316-2320.

Lever, J., Weaver, D., 1982. A theoretical model for fluid-elastic instability in heat exchanger tube bundles. J. of Pressure Vessel Tech. 104, 147-158.

Marcel, T., 2011. Simulation numérique et modélisation de l'interaction fluide-structure sous turbulence dans un faisceau de tubes soumis un coulement transverse nombre de Reynolds élevé. Thèse de doctorat, INPT (Institut National Polytechnique de Toulouse).

Martina, G., Braza, M., Hoarau, Y., Harran, G., 2008. Turbulence modelling of the ow past a pitching NACA0012 airfoil at $10^{5}$ and $10^{6}$ Reynolds numbers. J. Flds and Struct. (24), 1294-1303. 
Menter, F., 1994. Two-equation eddy-viscosity turbulence models for engineering applications. AIAA J. 32(8), 1598-1605.

Menter, F., Bender, R., 2003. A scale-adaptive simulation model for turbulent flow prediction. AIAA paper 2003-0767, Reno, NV.

Menter, F., Egorov, Y., 2005. A scale-adaptive simulation model using twoequation models. AIAA paper 2005-1095, Reno, NV.

Paidoussis, M. P., Price, S. J., 1988. The mechanisms underlying flow-induced instabilities of cylinder arrays in crossflow. J. of Fluid Mech. 187, 45-59.

Persillon, H., Braza, M., 1998. Physical analysis of the transition to turbulence in the wake of a circular cylinder by three-dimensional Navier-Stokes simulation. J. of Fluid Mech. 365, 23-88.

Shinde, V., Braza, M., Longatte, E., Baj, F., Hoarau, Y., Deloze, T., 2013. Numerical Simulation of Fluid-Structure Interaction in Square Normal Cylinder Arrays. In: ERCOFTAC International Symposium on Unsteady Separation in Fluid-Structure Interaction, Mykonos, Greece, June 17-21, 2013.

Skopek, M. G., Braza, M., Hoarau, Y., Thiele, F., 2012. Hybrid RANS-LES modeling of a strongly detached turbulent flow around a tandem cylinders configuration. In: S. Fu, W. Haase, S. P., Schwamborn, D. (Eds.), Progress in Hybrid RANS-LES Modelling, Notes on Numerical Fluid Mechanics and Multidisciplinary Design, Vol. 117. Springer-Verlag, Berlin, pp. 219-229.

Smagorinsky, J., 1963. General circulation experiments with the primitive equations. Month. Weather Rev. 93, 99-165.

Spalart, P., Allmaras, S., 1992. A one-equation turbulence model for aerodynamic flows. AIAA Paper 439.

Spalart, P., Deck, S., Shur, M., Squires, K., Strelets, M., Travin, A., 2006. A new version of detached-eddy simulation, resistant to ambiguous grid densities. Theor. Comput. Fluid Dyn. 20, 181-195.

Tanaka, H., Takahara, S., 1981. Fluid elastic vibration of tube array in cross flow. Journal of Vound and Vibration 77, 19-37. 
Tanaka, H., Takahara, S., 1982. Flow induced vibration of tube arrays with various pitch-to-diameter ratios. J. Pressure Vessel Technol. 104(3), 168174.

Vos, J., Chaput, E., Arlinger, B., Rizzi, A., Corjon, A., 1998. Recent advances in aerodynamics inside the NSMB (Navier-Stokes Multi-Block) consortium. AIAA Paper 0802.

Weaver, D., 2008. Some thoughts on the elusive mechanism of fluid elastic instability in heat exchanger tube arrays. flow-induced vibration. In: The 9th international conference on flow-induced vibration. Prague, Czech Republic.

Wu, S., Miau, J., Hu, C., Chou, J., 2005. On low-frequency modulations and three-dimensionality in vortex shedding behind a normal plate. J. Fluid Mech. (526), 117-146. 